\title{
Analysis of measurement systems mathematical models by using the comparison of functions
}

\author{
Ilkiv V. S., Nytrebych Z. M., Pukach P. Ya., Kohut I. V., Pakholok B. B. \\ Lviv Polytechnic National University, \\ 12 S. Bandera Str., 79013, Lviv, Ukraine
}

(Received 18 May 2019; Revised 10 October 2019; Accepted 10 October 2019)

\begin{abstract}
We propose an approach to mathematical modeling for a system on the basis of comparing scalar products in spaces of real functions integrable on the segment. This approach may be used in discrete and continuous measurement systems and some combinatorial systems, in which comparison problem of function exists in the process of synthesis and optimal choice of their parameters. In theory, such problems are characteristic of boundary value problems for equations of mathematical physics, in particular for multi-point problems that describe oscillatory processes in mechanisms. We have found the necessary and sufficient conditions for such a comparison. We use special transformations of sums and integrals that appear in the corresponding scalar products for vectors and functions.
\end{abstract}

Keywords: measurement systems, optimization, scalar product, integration by parts, integrable functions, polynomials.

2000 MSC: 26D15, 93A 30

UDC: $517,519.1,519.85$

DOI: $10.23939 / \mathrm{mmc} 2019.02 .268$

\section{Introduction}

Investigation of the behavior of systems of diverse nature and comparison of their quantitative and qualitative characteristics are the main goals of mathematical modeling. In this process, we use continuous or discrete parameters (functions) or continuous-discrete combinations of functions. Comparison of models implies a comparison of functions that could take various forms (comparison at points, comparison in the domains, in particular, comparison on the basis of scalar products that is proposed) depending on the nature of the function and its interpretation.

The general definition of the 'function' term in mathematics and natural sciences was formed by classical mathematics for several centuries on the basis of different approaches: geometric (through the function graph), analytical (as a mapping (relation) between sets), constructive (as a superposition of elementary functions). The class of functions was extended considerably in the twentieth century due to the notion of a generalized function (as a continuous linear functional on a set of smooth (test) functions). This type of construction often uses scalar products and their continuous extensions. The representation of functions in the form of series also usually implies a scalar product for calculating the coefficients of a series (Fourier coefficients). From that, we can conclude the method of constructing approximations to functions and their numerical characteristics.

The property of the equality of two generalized functions (the equality of a function to zero) in a given domain uses a set of test functions with an appropriate localization. A comparison of functions in the sense of order (greater-less) can also be made on the basis of scalar product and selected set of test functions.

The problem of ordering objects of a combinatorial type is characteristic of various discrete and distributed systems with control parameters. Here the scalar product is given on vectors with a finite or infinite number of components (coordinates) in the form of the sum (series) of products - Euclidean product.

In particular, the problem of ordering combinatorial objects often occurs in modelling systems of measurement of observed quantity [1, p. 230]. Such an arrangement arises when planning experiments on measuring complexes $[1,2]$. 
The theoretical study of solutions of problems with multipoint conditions for partial differential equations also leads to a comparison of scalar products of vectors formed by rearrangements of their components [3-8]. Selecting the greatest scalar product often means determining and evaluating the dominant harmonic of the solution, its qualitative and quantitative characteristics [9-11]. Similar questions may arise for equations of infinite order [12], operator equations [13-15], equations on a manifold [16], problems with Dirichlet conditions [17], with integral conditions [18], mixed problems [19].

In [20] we studied the problem of comparing vectors and specified the necessary and sufficient conditions for their comparability. Consequently, some vectors cannot be compared, and some can be.

Let there be given two vectors $\boldsymbol{x}=\left(x_{0}, x_{1}, \ldots, x_{n}\right)$ and $\boldsymbol{y}=\left(y_{0}, y_{1}, \ldots, y_{n}\right)$ from the space $\mathbb{R}^{n+1}$, at that $x_{0} \leqslant x_{1} \leqslant \cdots \leqslant x_{n}$ and $y_{0} \leqslant y_{1} \leqslant \cdots \leqslant y_{n}$, and the vectors $\boldsymbol{x}^{\alpha}=\left(x_{\alpha_{0}}, x_{\alpha_{1}}, \ldots, x_{\alpha_{n}}\right)$ and $\boldsymbol{y}^{\beta}=\left(y_{\beta_{0}}, y_{\beta_{1}}, \ldots, y_{\beta_{n}}\right)$, where $\alpha=\left(\alpha_{0}, \alpha_{1}, \ldots, \alpha_{n}\right)$ and $\beta=\left(\beta_{0}, \beta_{1}, \ldots, \beta_{n}\right)$ are permutations of the set of indexes $\{0,1, \ldots, n\}$.

Among the set of numbers $\left(\boldsymbol{x}^{\alpha}, \boldsymbol{y}^{\beta}\right)$, where $\left(\boldsymbol{x}^{\alpha}, \boldsymbol{y}^{\beta}\right)=\sum_{i=0}^{n} x_{\alpha_{i}} y_{\beta_{i}}$, there are not more than $(n+1)$ ! distinct ones, also there hold the inequalities

$$
\sum_{i=0}^{n} x_{i} y_{n-i} \leqslant\left(\boldsymbol{x}^{\alpha}, \boldsymbol{y}^{\beta}\right) \leqslant \sum_{i=0}^{n} x_{i} y_{i}=(\boldsymbol{x}, \boldsymbol{y}) .
$$

In particular, $\left(\boldsymbol{x}, \boldsymbol{y}^{(n, \ldots, 1,0)}\right) \leqslant\left(\boldsymbol{x}, \boldsymbol{y}^{\alpha}\right) \leqslant\left(\boldsymbol{x}, \boldsymbol{y}^{(0,1, \ldots, n)}\right)$, but for arbitrary fixed pair of permutations $\alpha$ and $\beta$ there could be either $\left(\boldsymbol{x}, \boldsymbol{y}^{\alpha}\right) \geqslant\left(\boldsymbol{x}, \boldsymbol{y}^{\beta}\right)$ or $\left(\boldsymbol{x}, \boldsymbol{y}^{\alpha}\right) \leqslant\left(\boldsymbol{x}, \boldsymbol{y}^{\beta}\right)$ depending on the vector $\boldsymbol{x}$.

The question arises on the possibility of comparison of vectors $\boldsymbol{y}^{\alpha}$ and $\boldsymbol{y}^{\beta}$ by the values of their scalar products not only for one fixed vector, but for the whole set of ordered vectors $\boldsymbol{x}$.

For arbitrary fixed permutations $\alpha$ and $\beta$, the inequality $\left(\boldsymbol{x}, \boldsymbol{y}^{\alpha}\right) \geqslant\left(\boldsymbol{x}, \boldsymbol{y}^{\beta}\right)$ holds on the set of ordered vectors $x \in \mathbb{R}^{n+1}$ if and only if there hold [20] the inequalities $\sum_{i=j}^{n} y_{\alpha_{i}} \geqslant \sum_{i=j}^{n} y_{\beta_{i}}$ for $j=1, \ldots, n$ (similar inequalities were established while comparing any pair $\boldsymbol{y}, \boldsymbol{z}$ of vectors from the space $\left.\mathbb{R}^{n+1}\right)$.

For $n=1$, those conditions are $y_{\alpha_{1}} \geqslant y_{\beta_{1}}$, so in this case for each pair $(\alpha, \beta)$ we have $\left(\boldsymbol{x}, \boldsymbol{y}^{\alpha}\right) \geqslant$ $\left(\boldsymbol{x}, \boldsymbol{y}^{\beta}\right)$ or $\left(\boldsymbol{x}, \boldsymbol{y}^{\alpha}\right) \leqslant\left(\boldsymbol{x}, \boldsymbol{y}^{\beta}\right)$ at once for all ordered $\boldsymbol{x} \in \mathbb{R}^{n+1}$, in particular, $\left(\boldsymbol{x}, \boldsymbol{y}^{\alpha}\right)=\left(\boldsymbol{x}, \boldsymbol{y}^{\beta}\right)+\left(x_{1}-\right.$ $\left.x_{0}\right)\left(y_{\alpha_{1}}-y_{\beta_{1}}\right)$.

For the case $n \geqslant 2$, that alternative does not hold. In fact, for $n=2$ and $y^{\alpha}=(2,3,1), y^{\beta}=(3,1,2)$ we calculate

$$
\left(\boldsymbol{x}, y^{\alpha}\right)=1 \cdot 2+5 \cdot 3+5 \cdot 1=22>18=1 \cdot 3+5 \cdot 1+5 \cdot 2=\left(\boldsymbol{x}, y^{\beta}\right),
$$

for $\boldsymbol{x}=(1,5,5)$ and for $\boldsymbol{x}=(1,2,8)$

$$
\left(\boldsymbol{x}, y^{\alpha}\right)=1 \cdot 2+2 \cdot 3+8 \cdot 1=16<21=1 \cdot 3+2 \cdot 1+8 \cdot 2=\left(\boldsymbol{x}, y^{\beta}\right) .
$$

Extending such results for comparing of functions $[a, b] \rightarrow \mathbb{R}$, where $a \in \mathbb{R}$ and $b \in \mathbb{R}$, is the topic of this paper. Instead of the method of summing by parts (see [20]), which is widely used in the theory of series [21-24], for numerical methods of solving boundary value problems for partial differential equations [25-29], we use the method of integrating by parts [18, 19, 24].

\section{Formulation of the problem and solvability}

For the segment $[a, b]$ of nonzero length, we denote: $\mathbf{R}(a, b)$ is a space of integrable on $[a, b]$ functions, $\mathbf{C d}^{1}(a, b)$ is a space of functions with piecewise-continuous on $[a, b]$ derivative, as well as the subset $\mathbf{C d}_{\leqslant}^{1}(a, b)$ of the set $\mathbf{C} \mathbf{d}^{1}(a, b)$, whose elements are functions with nonnegative on the segment $[a, b]$ derivative. This obviously implies that $\mathbf{C d}^{1}(a, b) \subset \mathbf{R}(a, b)$ and the piecewise-linear functions belong to $\mathbf{C d}^{1}(a, b)$. 
A scalar product in the space $\mathbf{R}(a, b)$ is defined by the formula

$$
(f, g)=\int_{a}^{b} f(x) g(x) d x .
$$

If $f \in \mathbf{C d}^{1}(a, b)$, then $d f=f^{\prime} d x$, where the derivative $f^{\prime}=f^{\prime}(x)$ is piecewise-continuous (hence, bounded) function $[a, b] \rightarrow \mathbb{R}$.

For arbitrary pair of functions $g=g(x), h=h(x)$ from the space $\mathbf{R}(a, b)$, we form one more pair of functions

$$
u=u(x)=\int_{x}^{b} g(t) d t, \quad v=v(x)=\int_{x}^{b} h(t) d t .
$$

Then those functions are uniformly continuous on $[a, b]$ generalized solutions of the following Cauchy problems:

$$
u^{\prime}=-g, \quad u(b)=0, \quad v^{\prime}=-h, \quad v(b)=0 .
$$

We study the question on finding the conditions for comparing functions $g$ and $h$ from the space $\mathbf{R}(a, b)$, i.e. holding of the inequality $(f, g) \geqslant(f, h)$ for all functions $f$ from the set $\mathbf{C d}_{\leqslant}^{1}(a, b)$.

Theorem 1. For given functions $g$ and $h$ from the space $\mathbf{R}(a, b)$ for all functions $f$ from the set $\mathbf{C d}_{\leqslant}^{1}(a, b)$ there hold the inequality

$$
(f, g) \geqslant(f, h)
$$

if and only if

$$
u(a)=v(a), \quad u(x) \geqslant v(x), \quad x \in[a, b] .
$$

Let the functions $g \in \mathbf{R}(a, b)$ and $h \in \mathbf{R}(a, b)$ satisfy conditions (5), then for arbitrary fixed function $f \in \mathbf{C d}_{\leqslant}^{1}(a, b)$ there holds the equality

$$
(f, g)=(f, h)+\int_{J} f^{\prime}(x)(u(x)-v(x)) d x,
$$

where $J=\left\{x \in[a, b]: f^{\prime}(x)(u(x)-v(x))>0\right\}$. Hence, if $J=\varnothing$, then $(f, g)=(f, h)$, and if $J \neq \varnothing$, then inequality (4) is strict; if

$$
J_{f}(\delta)=\left\{x \in[a, b]: f^{\prime}(x) \geqslant \delta\right\}, \quad J_{g, h}(\Delta)=\{x \in[a, b]:(u(x)-v(x)) \geqslant \Delta\},
$$

where $\delta>0$ and $\Delta>0$, then

$$
(f, g) \geqslant(f, h)+\delta \int_{J_{f}(\delta)}(u(x)-v(x)) d x, \quad(f, g) \geqslant(f, h)+\Delta \int_{J_{g, h}(\Delta)} f^{\prime}(x) d x .
$$

If $J_{f}(\delta)=[c, d] \subset[a, b]$, where $c<d$, then

$$
(f, g) \geqslant(f, h)+\delta \int_{c}^{d}(\min \{x, d\}-c)(g(x)-h(x)) d x
$$

in particular, $(f, g) \geqslant(f, h)+\delta \int_{a}^{b} x(g(x)-h(x)) d x$ in case when $[c, d]=[a, b]$, if $J_{g, h}(\delta)=[c, d]$, then $(f, g) \geqslant(f, h)+\Delta \cdot(f(d)-f(c))$.

Proof. For the scalar product $(f, g)$ we will use the formula for integrating by parts and formulas (1)(3), then

$$
(f, g)=-\int_{a}^{b} f(x) u^{\prime}(x) d x=f(a) u(a)+\int_{a}^{b} f^{\prime}(x) u(x) d x=f(a) u(a)+\left(f^{\prime}, u\right) .
$$

Mathematical Modeling and Computing, Vol. 6, No. 2, pp. 268-275 (2019) 
Similarly, we obtain the formula $(f, h)=f(a) v(a)+\left(f^{\prime}, v\right)$ and the formula for the difference

$$
(f, g)-(f, h)=(f, g-h)=f(a)(u(a)-v(a))+\left(f^{\prime}, u-v\right) .
$$

If conditions (5) are satisfied, then $(f, g)-(f, h)=\int_{a}^{b} f^{\prime}(x)(u(x)-v(x)) d x$ and $f^{\prime}(x)(u(x)-v(x)) \geqslant 0$ for $x \in[a, b]$ and $f \in \mathbf{C d}_{\leqslant}^{1}(a, b)$. This implies inequality (4). Therefore, conditions (5) are sufficient conditions.

Vice versa, if the function $f$ is constant, i.e. $f=f(a)$ and $f^{\prime}=0$, then by formula (8) we obtain

$$
(f, g)-(f, h)=(f, g-h)=f(a)(u(a)-v(a)) \geqslant 0 .
$$

This implies the necessity of the equality $u(a)=v(a)$. Let the second condition (5) be not satisfied, then the uniform continuousness of the functions $u$ and $v$ implies that $u(x)+w<v(x)$ on a certain segment $[c, d] \subset[a, b]$, where $a<c<d<b, w$ is a certain positive constant. For the function $f \in \mathbf{C d}_{\leqslant}^{1}(a, b)$ of the form $f(x)=f(a)$ on $[a, c], f(x)=f(a)-c+k x$ on $[c, d]$ and $f(x)=f(a)-c+d$ on $[d, b]$ we have $(f, g)-(f, h)=k \int_{c}^{d}(u(x)-v(x)) d x-<k w(d-c)<0$, where $k$ is a certain positive constant. Therefore, inequality (4) does not hold; hence, we proved the necessity of conditions (5).

Let the functions $g \in \mathbf{R}(a, b)$ and $h \in \mathbf{R}(a, b)$ satisfy conditions (5), then formula (6) follows from equality (8). If $J \neq \varnothing$, then in that formula, the integral has a positive value and $(f, g)>(f, h)$, otherwise we obtain the equality $(f, g)=(f, h)$.

Formula (7) also follows from equality (8) and the definition of the sets $J_{f}(\delta)$ and $J_{g, h}(\Delta)$. If among those sets there is the segment $[c, d]$, then appropriate calculation of the integrals in formula (7) leads to the equalities: $\int_{c}^{d} f^{\prime}(x) d x=f(d)-f(c)$,

$$
\begin{aligned}
\int_{c}^{d}(u(x)-v(x)) d x=\int_{c}^{d} d x \int_{x}^{b} & (g(t)-h(t)) d t=\int_{c}^{d} d t \int_{c}^{t}(g(t)-h(t)) d x+ \\
& \quad+\int_{d}^{b} d t \int_{c}^{d}(g(t)-h(t)) d x=\int_{c}^{b}(\min \{t, d\}-c)(g(t)-h(t)) d t .
\end{aligned}
$$

In case if $d=b$ we have $\min \{t, d\}=t$ and $\int_{c}^{b}(u(x)-v(x)) d x=\int_{c}^{b}(t-c)(g(t)-h(t)) d t$. If also $c=a$, then $\int_{a}^{b}(g(t)-h(t)) d t=u(a)-v(a)=0$. This proves our theorem.

Remark 1. If the functions $g$ and $h$ satisfy conditions (5) and $g \neq h$, then the difference $g-h$ takes on the segment $[a, b]$ positive and negative values, moreover, the areas between the graphs of those functions are the same above the graph and below the graph of each one.

\section{Numerical and analytical examples}

We give two examples for illustration of general results obtained above. In the first one, we compared on the segment $[1,2] \subset \mathbb{R}$ the logarithmic and the exponential functions. In the second example, we compared scalar products for arbitrary degree polynomials.

Example 1. Let $g(x)=e^{x-1} /(1-e)$ and $h(x)=\ln x /(1-\ln 4)$, then on the segment $[1,2]$ those functions are continuous (see Fig. 1), i.e. $g \in \mathbf{R}(1,2)$ and $h \in \mathbf{R}(1,2)$.

Let us check the condition (5). Since

$$
u(x)=\int_{x}^{2} \frac{e^{x-1}}{1-e} d x=\frac{e^{x-1}-e}{e-1}, \quad v(x)=\int_{x}^{2} \frac{\ln x}{1-\ln 4} d x=\frac{x(\ln x-1)-\ln 4+2}{\ln 4-1}
$$

and $(u-v)^{\prime}=h-g$, then $u(1)=v(1)=-1$ and the function $h-g$ has only one zero. That is the result of strictly monotonic decreasing of the functions $g$ and $h$ and of the different signs of the difference 
$h-g$ at the bounds of the segment $[1,2]$ :

$$
h(1)-g(1)=1 /(e-1)>0, \quad h(2)-g(2)=\ln 2 /(1-\ln 4)+e /(e-1)<-1 / 5<0 .
$$

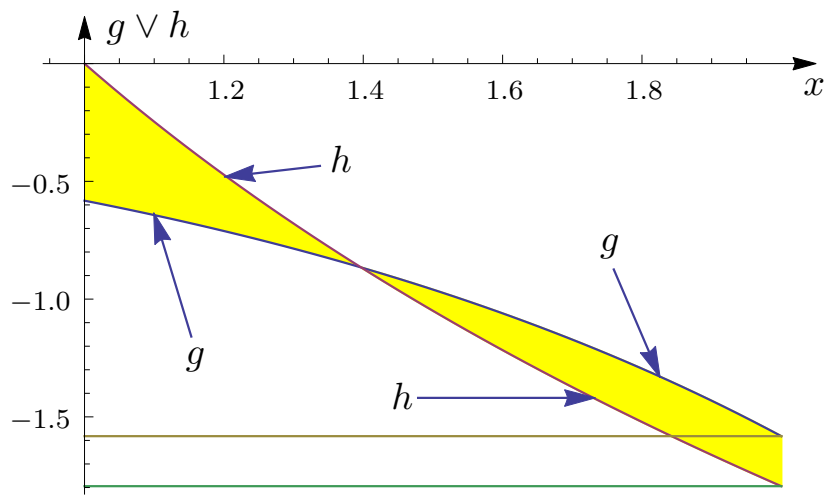

Fig. 1. Graphs of the functions $g(x)=e^{x-1} /(1-e)$ and $h(x)=\ln x /(1-\ln 4)$.

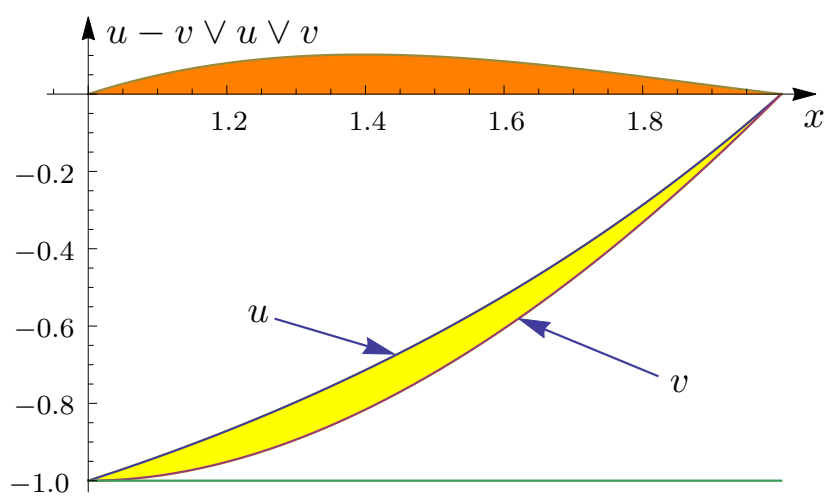

Fig. 2. Graphs of the functions $u(x)=\left(e^{x-1}-e\right) /(e-$ $1), v(x)=(x(\ln x-1)-\ln 4+2) /(\ln 4-1)$ and $u-v$.

Therefore, the function $u-v$ has a positive maximum at the point $x_{0} \in(1,2)$ for which $h\left(x_{0}\right)=g\left(x_{0}\right)$. Hence, on the whole interval $(1,2)$ there holds (see Fig. 2$)$ the inequality $u(x) \geqslant v(x)$. So, the function $e^{x-1} /(1-e)$ is comparable with the function $\ln x /(1-\ln 4)$, namely the first one is "greater than" the second one.

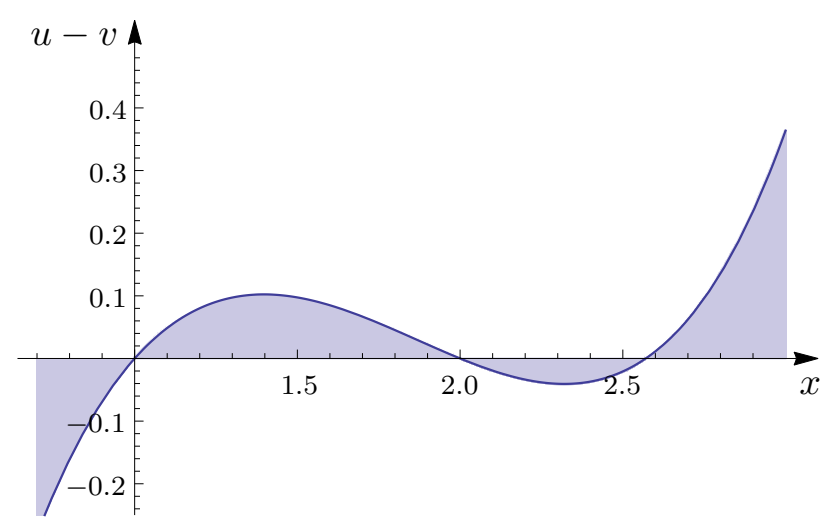

Fig. 3. Graph of the function $u-v$ in the variable $x$.



Fig. 4. Graphs of the polynomials $t^{5},(1-t) t^{s-1}$, where $s=1, \ldots, 5$.

On the segment $\left[2, x^{*}\right]$, where $x^{*} \approx 2.57$ is the last (the greatest) zero of the function $u-v$, vice versa (see Fig. 3), the function $e^{x-1} /(1-e)$ is "less than" the function $\ln x /(1-\ln 4)$.

Example 2. Let $g$ and $h$ be polynomials of degree not higher than $n$ on the segment $[a, b]$, then

$$
g(x)=g(a)+\sum_{j=1}^{n} g^{(j)}(a) \frac{(x-a)^{j}}{j !}, \quad h(x)=h(a)+\sum_{j=1}^{n} h^{(j)}(a) \frac{(x-a)^{j}}{j !} .
$$

Denoting $t=\frac{x-a}{b-a}, g_{j}=g^{(j)}(a) \frac{(b-a)^{j}}{j !}, h_{j}=h^{(j)}(a) \frac{(b-a)^{j}}{j !}$, where $j=1, \ldots, n$, we obtain

$$
g(x)=g_{0}+\sum_{j=1}^{n} g_{j}\left(t^{j}-\frac{1}{j+1}\right), \quad h(x)=h_{0}+\sum_{j=1}^{n} h_{j}\left(t^{j}-\frac{1}{j+1}\right)
$$

for $g_{0}=g(a)+\sum_{j=1}^{n} \frac{g_{j}}{j+1}$ and $h_{0}=h(a)+\sum_{j=1}^{n} \frac{h_{j}}{j+1}$. At that $0 \leqslant t \leqslant 1,1-t=\frac{b-x}{b-a}$ and $d x=(b-a) d t$. 
Now we find the functions $u$ and $v$ :

$$
u(x)=(b-x) g_{0}+(b-a) \sum_{j=1}^{n} \frac{g_{j}}{j+1} \int_{x}^{b}\left((j+1) t^{j}-1\right) d t=(b-x) g_{0}+(b-a) t(1-t) \sum_{j=1}^{n} \frac{g_{j}}{j+1} \sum_{s=1}^{j} t^{s-1} .
$$

From that we obtain the polynomials

$$
\begin{aligned}
& u(x)=(b-x) g_{0}+\frac{(x-a)(b-x)}{b-a} \sum_{j=1}^{n} \frac{g_{j}}{j+1} \sum_{s=1}^{j} t^{s-1}, \\
& v(x)=(b-x) h_{0}+\frac{(x-a)(b-x)}{b-a} \sum_{j=1}^{n} \frac{h_{j}}{j+1} \sum_{s=1}^{j} t^{s-1} .
\end{aligned}
$$

For which $u(a)=(b-a) g_{0}, v(a)=(b-a) h_{0}$. Thus by condition (5) we have $g_{0}=h_{0}$ and

$$
u(x)-v(x)=\frac{(x-a)(b-x)}{b-a} \sum_{j=1}^{n} \frac{g_{j}-h_{j}}{j+1} \sum_{s=1}^{j} t^{s-1} \geqslant 0
$$

on the segment $[a, b]$. If $g_{j} \geqslant h_{j}$ for $j=1, \ldots, n$, then inequality (9) holds and is strict on the interval $(a, b)$, if $g_{j}>h_{j}$ at least for one $j$. If we rewrite the difference $u-v$ in the form

$$
u(x)-v(x)=\frac{(x-a)(b-x)}{b-a} \sum_{s=1}^{n}\left(u_{s}-v_{s}\right) t^{s-1},
$$

where $u_{s}=\sum_{j=s}^{n} \frac{g_{j}}{j+1}, v_{s}=\sum_{j=s}^{n} \frac{h_{j}}{j+1}$, then inequality (9) holds under the condition $u_{s} \geqslant v_{s}$, $s=1, \ldots, n$, and is strict on $(a, b)$, if $u_{s}>v_{s}$ at least for one $s$. If we rewrite inequality (9) one more time

$$
u(x)-v(x)=\frac{(x-a)(b-x)}{b-a}\left((1-t) \sum_{s=1}^{n-1} \sum_{l=1}^{s}\left(u_{l}-v_{l}\right) t^{s-1}+\sum_{l=1}^{n}\left(u_{l}-v_{l}\right) t^{n-1}\right),
$$

then we obtain one more sufficient condition for comparing the polynomials $g$ and $h$ :

$$
\sum_{l=1}^{s} u_{l} \geqslant \sum_{l=1}^{s} v_{l}, \quad s=1, \ldots, n .
$$

The graphs of the positive on $(0,1)$ polynomials $t^{n-1}$ and $(1-t) t^{s-1}$, where $s=1, \ldots, n-1$, are given in Fig. 4 for $n=6$.

Condition (10) is the weakest among the written sufficient conditions, whereas the strongest one is the condition $g_{j} \geqslant h_{j}, j=1, \ldots, n$, in formula (9), which implies all the next conditions.

\section{Conclusions}

We obtain necessary and sufficient conditions on the given (fixed) functions $g$ and $h$, which are integrable on the segment $[a, b]$, under which for arbitrary functions $f$ with nonnegative piecewisecontinuous derivative, the scalar product $(f, g)$ does not exceed the scalar product $(f, h)$. Those conditions are written in the form of inequalities, which are easy to check. We also give the illustrative (numerical and analytical) examples of applications of the conditions obtained. These results can be used to synthesize and optimize continuous and discrete parameters of signal measurement systems by analyzing their mathematical models. 
[1] Pyt'ev J. P. Mathematical methods of interpretation of experiment. Moscow, High School (1989), (in Russian).

[2] Kozlov A. A., Pyt'yev Yu. P. The efficiency of reduction of measurements and some problems of meteringcomputing assembly design. USSR Computational Mathematics and Mathematical Physics. 27 (2), 1-6 (1987).

[3] Il'kiv V. S., Ptashnyk B. I. An ill-posed nonlocal two-point problem for systems of partial differential equations. Sib. Math. Journ. 46 (1), 94-102 (2005).

[4] Nytrebych Z. M., Malanchuk O. M., Il'kiv V.S., Pukach P. Ya. Homogeneous problem with two-point conditions in time for some equations of mathematical physics. Azerb. Journ. of Math. 7 (2), 180-196 (2017).

[5] Nytrebych Z., Malanchuk O., Il'kiv V., Pukach P. On the solvability of two-point in time problem for PDE. Italian J. of Pure and Appl. Math. 38, 715-726 (2017).

[6] Ptashnyk B. I., Symotyuk M. M. Multipoint Problem for Nonisotropic Partial Differential Equations with Constant Coefficients. Ukr. Math. Journ. 55 (2), 293-310 (2003).

[7] Vasylyshyn P. B., Klyus I. S., Ptashnyk B. Yo. Multipoint problem for linear equations with variable coefficients. Ukr. Math. Journ. 48 (11), 1659-1668 (1996).

[8] Vasylyshyn P. B., Savka I. Ya., Klyus I. S. Multipoint nonlocal problem for factorized equation with dependent coefficients in conditions. Carpath. Math. Publ. 7 (1), 22-27 (2015).

[9] Horodetskyi V., Martynyuk O., Petryshyn R. Correct solvability of a nonlocal multipoint in time problem for one class of evolutionary equations. Ukr. Math. Journ. 65 (3), 377-392 (2013).

[10] Il'kiv V.S. Incorrect nonlocal boundary value problem for partial differential equations. Func. Analysis and its applications. 197, 115--121 (2004).

[11] Il'kiv V.S., Ptashnyk B. I. Problems for partial differential equations with nonlocal conditions. Metric approach to the problem of small denominators. Ukr. Math. Journ. 58 (12), 1847-1875 (2006).

[12] Il'kiv V.S. A nonlocal boundary-value problem for partial differential equations of infinite order. Ukr. Math. Journ. 35 (4), 420-423 (1983).

[13] Il'kiv V. S., Strap N. I. Solvability of the Nonlocal Boundary-Value Problem for a System of DifferentialOperator Equations in the Sobolev Scale of Spaces and in a Refined Scale. Ukr. Math. Journ. 67 (5), 690-710 (2015).

[14] Il'kiv V. S., Strap N. I. Solvability of a nonlocal boundary-value problem for the operator-differential equation with weak nonlinearity in a refined scale of Sobolev spaces. Journ. of Math. Scien. 218 (1), 1-15 (2016).

[15] Il'kiv V. S., Strap N. I. Nonlocal Boundary-Value Problem for a Differential-Operator Equation with Weak Nonlinearity in the Spaces of Dirichlet-Taylor Series with Fixed Spectrum. Journ. of Math. Scien. 231 (4), 572-585 (2018).

[16] Savka I. Ya. A nonlocal boundary-value problem for partial differential equations with constant coefficients belonging to smooth curves. Journ. of Math. Scien. 174 (2), 136-158 (2011).

[17] Nytrebych Z., Il'kiv V., Pukach P., Malanchuk O. On nontrivial solutions of homogeneous Dirichlet problem for partial differential equation in a layer. Krag. J. of Math. 42 (2), 193-207 (2018).

[18] Il'kiv V.S., Nytrebych Z. M., Pukach P. Ya. Nonlocal problem with moment conditions for hyperbolic equations. Electron. J. Differential Equations. 2017 (265), 1-9 (2017).

[19] Pukach P., Il'kiv V., Nytrebych Z., Vovk M. On nonexistence of global in time solution for a mixed problem for a nonlinear evolution equation with memory generalizing the Voigt-Kelvin rheological model. Opuscula Math. 35 (5), 735-753 (2017).

[20] Il'kiv V., Nytrebych Z., Pukach P., Kohut I., Pacholok B. Order Relation on Scalar Products in Real Linear Spaces. 2019 IEEE 15th International Conference on the Experience of Designing and Application of CAD Systems (CADSM). Proceedings. No. 8779356, 32-35 (2019).

[21] Andrews G., Askey R., Roy R. Special Functions. Cambridge, Cambridge University Press (1999).

[22] Chu W. Abel's lemma on summation by parts and basic hypergeometric series. Advances in Applied Mathematics. 39 (4), 490-514 (2007). 
[23] Gasper G. Summation, transformation, and expansion formulas for bibasic series. Trans. Amer. Math. Soc. 312 (1), 257-277 (1989).

[24] Mosig J. R., Alvarez Melcon A. The summation-by-parts algorithm-a new efficient technique for the rapid calculation of certain series arising in shielded planar structures. IEEE Transactions on Microwave Theory and Techniques. 50 (1), 215-218 (2002).

[25] Gessel I. Finding identities with the WZ method. J. Symbolic Comput. 20 (5-6), 537-566 (1995).

[26] Kreiss H.-O., Scherer G. Finite element and finite difference methods for hyperbolic partial differential equations. Mathematical Aspects of Finite Elements in Partial Differential Equations. Proceedings of a Symposium Conducted by the Mathematics Research Center, the University of Wisconsin-Madison, April 1-3, 1974. 195-212 (1974).

[27] Strand B. Summation by parts for finite difference approximations for $\mathrm{d} / \mathrm{dx}$. Journal of Computational Physics. 110 (1), 47-67 (1994).

[28] Svärd M. On coordinate transformations for summation-by-parts operators. Journal of Scientific Computing. 20 (1), 29-42 (2004).

[29] Tkachov F. V. Algebraic algorithms for multiloop calculations The first 15 years. What's next? Nucl. Instr. and Methods in Phys. Research. Sect. A: Accel., Spectromet., Detect. and Assoc. Equip. 389 (1), 309-313 (1997).

\title{
Аналіз математичних моделей вимірювальних систем з використанням порівняння функцій
}

\author{
Ільків В. С., Нитребич З. М., Пукач П. Я., Когут І. В., Пахолок Б. Б. \\ Національний університет “Львівсъка політехніка", \\ вул. С. Бандери, 12, Львів, 79013, Україна
}

\begin{abstract}
У роботі запропоновано підхід до математичного моделювання систем на основі порівняння скалярних добутків у просторах, інтегровних на відрізку дійсних функцій. Цей підхід можна використовувати у дискретних і неперервних системах вимірювання та деяких комбінаторних системах у процесі синтезу та оптимального вибору їх параметрів, де постає задача порівняння функцій. Теоретично такі задачі характерні також для крайових задач для рівнянь математичної фізики, зокрема для багатоточкових задач, які описують коливні процеси у механізмах. Знайдено необхідні й достатні умови такого порівняння. Використано спеціальні перетворення сум та інтегралів, які входять у відповідні скалярні добутки для векторів та функцій.
\end{abstract}

Ключові слова: системи вимірювання, оптимізачія, скалярний добуток, інтегрування частинами, інтегровні функиї, многочлени.

2000 MSC: 26D15, 93A30

УДК: $517,519.1,519.85$ 